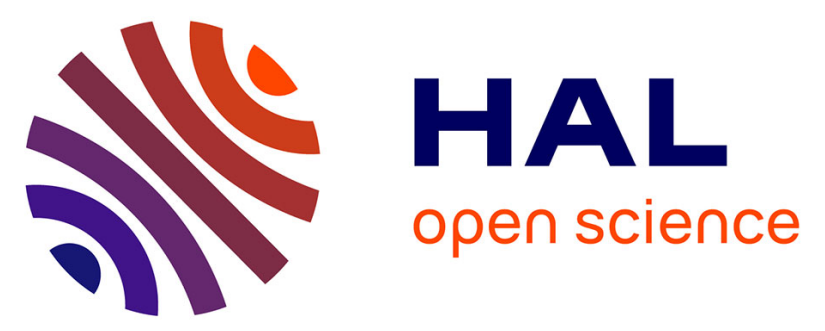

\title{
An electron density based analysis to establish the electronic adiabaticity of proton coupled electron transfer reactions
}

Ilaria Ciofini, Maria Gabriella Chiariello, | Federico Coppola, Fulvio Perrella, Marika Savarese, | Ilaria Ciofini, Umberto Raucci, Nadia Rega

\section{To cite this version:}

Ilaria Ciofini, Maria Gabriella Chiariello, | Federico Coppola, Fulvio Perrella, Marika Savarese, et al.. An electron density based analysis to establish the electronic adiabaticity of proton coupled electron transfer reactions. Journal of Computational Chemistry, 2020, 10.1002/jcc.26224 . hal-02879687

\section{HAL Id: hal-02879687 https://hal.science/hal-02879687}

Submitted on 12 Nov 2020

HAL is a multi-disciplinary open access archive for the deposit and dissemination of scientific research documents, whether they are published or not. The documents may come from teaching and research institutions in France or abroad, or from public or private research centers.
L'archive ouverte pluridisciplinaire HAL, est destinée au dépôt et à la diffusion de documents scientifiques de niveau recherche, publiés ou non, émanant des établissements d'enseignement et de recherche français ou étrangers, des laboratoires publics ou privés. 


\title{
An Electron Density Based Analysis to Establish the Electronic Adiabaticity of Proton Coupled Electron Transfer Reactions
}

\author{
Umberto Raucci $^{a}$, Maria Gabriella Chiariello ${ }^{a}$, Federico Coppola ${ }^{a}$, \\ Fulvio Perrella ${ }^{a}$, Marika Savarese ${ }^{b}$, Ilaria Ciofini ${ }^{c}$, Nadia Rega $^{a, d}$
}

April 21, 2020

\begin{abstract}
Electrons and protons are the main actors in play in Proton Coupled Electron Transfer (PCET) reactions, which are fundamental in many biological (i.e. photosynthesis and enzymatic reactions) and electrochemical processes. The mechanism, energetics and kinetics of PCET reactions are strongly controlled by the coupling between the transferred electrons and protons. Concerted PCET reactions are classified according to the electronical adiabaticity degree of the process. To discriminate among different mechanisms, we propose a new analysis based on the use of electron density based indexes. We choose, as test case, the 3-Methylphenoxyl/phenol system in two different conformations to show how the proposed analysis is a suitable tool to discriminate between the different degree of adiabaticity of PCET processes. The very low computational cost of this procedure is extremely promising to analyze and provide evidences of PCET mechanisms ruling the reactivity of many biological and catalytic systems.
\end{abstract}

Keywords: Density based indexes, Proton Coupled Electron Transfer, Electronic Adiabaticity Degree, Non Adiabatic Coupling, Density Functional Theory

a) Dipartimento di Scienze Chimiche, Università di Napoli 'Federico II', Complesso Universitario di M.S.Angelo, via Cintia, Napoli 80126, Italy

b) CompuNet, Istituto Italiano di Tecnologia, via Morego 30, I-16163 Genoa, Italy

c) Chimie ParisTech, PSL Research University, CNRS, Institute of Chemistry for Life and Health Sciences, 11 rue Pierre et Marie Curie, F-75005 Paris, France

d) CRIB Center for Advanced Biomaterials for Healthcare, Piazzale Tecchio, Napoli 80125, Italy

Correspondence to: Umberto Raucci, umberto.raucci@unina.it, Nadia Rega, nadia.rega@unina.it 


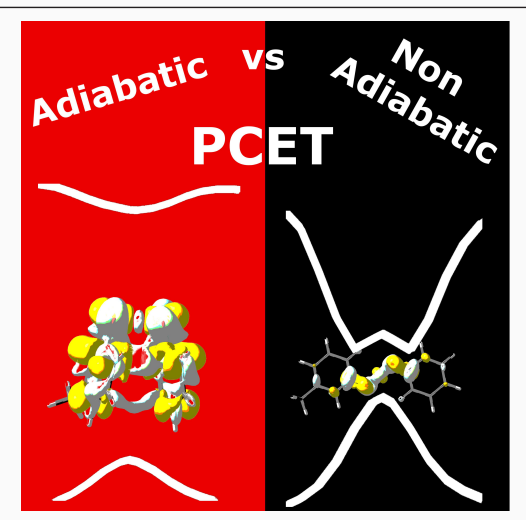

Concerted Proton Coupled Electron Transfer (PCET) reactions are classified according to the electronical adiabaticity degree of the process. We propose a new analysis based on the use of electron density based indexes to discriminate between different PCET mechanisms. Our analysis opens a new scenario for ab-initio simulations to investigate complex PCET mechanisms at very low computational cost. 


\section{Introduction}

Proton Coupled Electron Transfer (PCET) reactions are at the origin of many fundamental

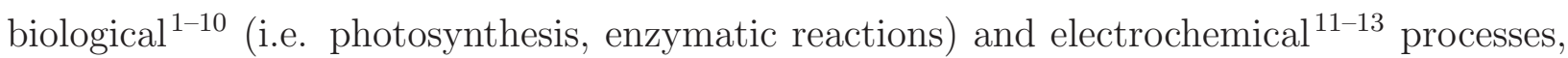
thanks to their versatile nature. The transfer of electrons and protons represents the leitmotif of PCET. ${ }^{14-19}$

The general PCET definition covers several different mechanisms and kinetics, controlled by the coupling between the transferred electrons and protons. In the last few years, many efforts were devoted to provide an universal classification of the complex family of PCET reactions. ${ }^{15,18-27}$ This is essential for supporting the interpretation of experimental data and also to drive the design of efficient charge transfer molecular machines suitable for catalysis and artificial photosynthesis. ${ }^{28-34}$

The classification of the PCET reactions proposed by Hammes Shiffer relies on the electronic, vibrational and vibronic adiabaticity degree between the transferred electron and proton. ${ }^{20-23}$ Concerted PCET reactions, where no stable intermediates are involved, are basically distinguished as Hydrogen Atom Transfer (HAT) or Electron Proton Transfer (EPT), depending on the electronic adiabaticity degree of the process. ${ }^{23}$

In a HAT process the reaction evolves only in the ground electronic state and it is electronically adiabatic. In this case the vertical gap between the ground and the first excited state is larger than the thermal energy. Otherwise, if the ground and the first excited state are strongly coupled together the reaction is electronically non-adiabatic. EPT reactions fall into this category. An adiabaticity parameter, defined as the ratio of the proton tunneling time and the electronic transition time, was introduced and widely adopted to distinguish between HAT and EPT mechanisms. ${ }^{20,21,35,36}$ Moreover, considering that the electronic adiabaticity is strictly related to the charge redistribution along the proton transfer coordinate, several diagnostic analyses were also performed. ${ }^{36,37}$ For example, the adiabaticity degree of PCET processes was evaluated analyzing the dipole moment variation along the reaction coordinate. ${ }^{36,37}$ Another less intuitive and more computational demanding analysis, based on the evaluation of the non-adiabatic coupling (NAC) vectors between the ground and the excited electronic state, was also proposed. ${ }^{36,37}$ The first order non-adiabatic coupling ma- 
trix elements between the $\mid \Psi_{m}>$ and $\mid \Psi_{n}>$ electronic states can be written as follows: ${ }^{38-40}$ $\tau_{m n}^{\xi}=\frac{\left\langle\Psi_{m}\left|\frac{\partial}{\partial \xi} \hat{V}_{n e}\right| \Psi_{n}\right\rangle}{E_{n}-E_{m}}$ where $\xi$ represents a Cartesian nuclear coordinate, $\hat{V}_{n e}$ is the electronnucleus attraction operator, while $\mathrm{E}_{m}$ and $\mathrm{E}_{n}$ are the energy eigenvalues of $\left|\Psi_{m}\right\rangle$ and | $\Psi_{n}>$, respectively.

The computation of NAC vectors is crucial to characterize regions of the Potential Energy Surface (PES) where two or more electronic states become degenerate (i.e. conical intersection points), and to follow the excited state non-adiabatic dynamics of the system. ${ }^{40-55}$

In the case of PCET reactions, large magnitude of the NAC vectors and a drastic change in the dipole moment along the reaction coordinate are observed for non-adiabatic reactions, whereas adiabatic processes show small magnitude of NAC and a slight variation of the dipole moment. ${ }^{36,37}$

In this context, the classification of PCET processes in terms of electronic adiabaticity would greatly benefit from a suitable tool able to condensate both the information of non-adiabatic coupling and dipole moment variation. These properties are intrinsically related to the ground and the excited state electron density distributions. Thus, we propose here an electron density based analysis to discriminate among concerted PCET reactions belonging to different adiabaticity regimes.

In detail, the $\mathrm{D}_{C T}$ electron density based index ${ }^{56,57}$ was employed as key instrument to quantify the ground-excited state electronic reorganization occuring during the PCET process. This index simply represents the distance between the barycenters of charge distributions associated respectively to the increase and the decrease of the electron density upon a vertical excitation. ${ }^{57}$

Recently, it was successfully applied to analyze excited state reactivity (i.e. excited state proton transfer reactions) in combination with both static and dynamical approaches. ${ }^{58-66}$ The information on the charge rearrangement after the vertical excitation is innate in the $\mathrm{D}_{C T}$ index, which is related to the ground-excited state dipole moments variation by construction. ${ }^{57}$ Moreover, it allows to quantify the electron density redistribution between two electronic states, containing information indirectly related to the non-adiabatic coupling. PES regions characterized by a strong electronic coupling are expected to show an high similarity between the ground and the excited state electron densities. In these points the 
ground-first excited state electronic reorganization is small, corresponding to low values of $\mathrm{D}_{C T}$. The opposite behavior is expected in electronically uncoupled regions of the PES. Relying only on the evaluation of the ground and the excited state electron densities, the $\mathrm{D}_{C T}$ index is cheaply computed compared to the time-demanding NAC computation. Most importantly, it can be directly evaluated and used in combination to any quantum chemical method, such as DFT or post-Hartree Fock based. ${ }^{67}$

In this paper, this density based index was applied to discriminate the different adiabaticity degree in the PCET reaction of a prototype system, the 3-Methylphenoxyl/phenol one, hereafter named mMphenoxyl/phenol. For a related system, i.e. the phenoxyl/phenol dimer, a recent study of Harshan and co-workers showed that, depending on the geometrical arrangement of the two aromatic rings, the PCET reaction takes place with a HAT or an EPT mechanism. ${ }^{35}$ For this system the existence of two transition states corresponding to an open and a stacked geometry was proved, as well as the fact that the PCET reaction is electronically non-adiabatic for the open conformation, while it can be classified as electronic adiabatic reaction in the case of the stacked geometry. ${ }^{35}$

We simulated the reaction pathway for both the open and the stacked configurations of the mMphenoxyl/phenol dimer by the integration of the intrinsic reaction coordinate at density functional theory (DFT) level. ${ }^{68}$ The ground and first excited state PESs and their electron densities were also computed at DFT and TD-DFT ${ }^{69,70}$ level of theory.

The $\mathrm{D}_{C T}$ analysis along the reaction coordinate, suggested that this index provides a fairy picture of the degree of adiabaticity for the examined cases. A very nice agreement with the conclusions reported by Harshan and co-workers was also found.

\section{Methods}

The proton exchange reaction of the mMphenoxyl/phenol system was investigated at DFT and TD-DFT levels of theory. ${ }^{68-70}$ More in details, the M06-2X ${ }^{71} / 6-311+\mathrm{G}(\mathrm{d}, \mathrm{p})$ level was employed to locate the ground state transition state (TS) for both the open and the stacked conformations. The TS nature was confirmed by a subsequent frequency calculations (see 
Figure S1 for the displacement vectors along the normal mode with immaginary frequency). A completely planar TS could not be optimized for the open arrangement, therefore the coplanarity between the phenyl rings was enforced constraining the $\mathrm{CC}_{O M}-\mathrm{C}_{O} \mathrm{C}$ dihedral angle to be planar $\left(\mathrm{C}_{O M}\right.$ and $\mathrm{C}_{O}$ are the carbon atoms bound to $\mathrm{O}_{M}$ and $\mathrm{O}$, respectively, Figure 1). The reaction path was, then, followed by integrating the intrinsic reaction coordinate (IRC) at the M06-2X/6-311+G(d,p) level, employing the Hessian-based Predictor Corrector integrator. ${ }^{72-74}$ On the IRC structures single point calculations at CAM-B3LYP ${ }^{75} / 6-311+\mathrm{G}(\mathrm{d}, \mathrm{p})$ and TD-CAM-B3LYP/6-311+G(d,p) level of theory were performed to define the ground and first excited energy profiles. The M06-2X functional was employed for the geometry optimizations because it includes dispersion effects, whereas excited state properties were evaluated using the CAM-B3LYP functional that provides a robust and reliable description of the charge transfer excitation. ${ }^{76}$ Cartesian non adiabatic coupling vectors between the ground and first excited state were analitically computed according to the Refs. ${ }^{38,40}$ as implemented in the Gaussian package. ${ }^{77}$ The $\mathrm{D}_{C T}$ index was computed using relaxed densities. All calculations were performed using Gaussian 16 suite programs. ${ }^{77}$

\section{Open}
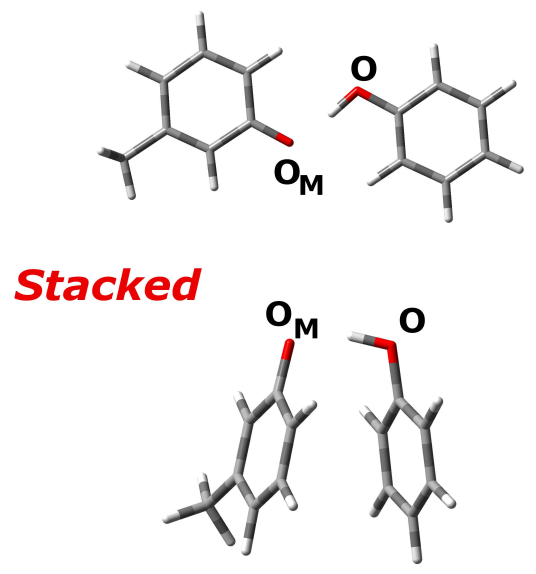

Figure 1: Schematic view and labelling of open and stacked conformations considered for reactants of the mMphenoxyl/phenol PCET reaction. 


\section{Results and Discussion}

The structural and energetic features of the PCET reactions are discussed in the next section, while the electron density based analysis is disclosed in section 3.2.

\subsection{Structural and energetic features}

Before describing in detail the electron density based analysis, we briefly illustrate structural and energetic features of the mMphenoxyl/phenol system, in both the open and stacked conformations. The main structural parameters of reactants, transition states and products are provided in Table S1 of the Supplementary Information (SI). The open geometry shows a planar arrangement of the two aromatic rings, with an $\mathrm{O}-\mathrm{H}-\mathrm{O}_{M}$ angle of $180^{\circ}$ at the TS structure, as illustrated in Figure 1. On the other hand, the stacked TS is characterized by a $\mathrm{O}-\mathrm{H}-\mathrm{O}_{M}$ angle of $166^{\circ}$. The ground and first excited PESs obtained by integrating the intrinsic reaction coordinate (IRC) in the ground state are reported in Figure 2 for the open and stacked geometries. In both cases the proton exchange reaction proceeds with a barrier: about $13 \mathrm{kcal} / \mathrm{mol}$ is the barrier computed for the open form, while a lower value (about $8 \mathrm{kcal} / \mathrm{mol}$ ) is found for the stacked conformation. Reactants and products are almost isoenergetic, although the proton is bound to the phenolic ring in a slightly more stable way.

The energy differences between the ground and first excited state surfaces are reported in Table S2 for reactants, transition states and products. In the case of the open geometry, the two surfaces are very close in energy near the transition state region (the vertical spacing at the TS is about $5 \mathrm{kcal} / \mathrm{mol}$ ). On the other hand, they are well separated in the stacked conformation, with an energy difference of about $27 \mathrm{kcal} / \mathrm{mol}$ at the TS. These results obtained at CAM-B3LYP 75 and TD-CAM-B3LYP levels of theory on the mMphenoxylphenol system are in very nice agreement with both the CASSCF and CDFT-CI calculations reported by Harshan and co-workers on the phenoxyl-phenol couple. ${ }^{35}$ Moreover, the CAMB3LYP energy profiles reproduce the different regimes of adiabaticity recognized for the two geometries. Indeed, an electronically non-adiabatic process occurs in the case of the open conformation, while an electronically adiabatic reaction is observed for the stacked one. 

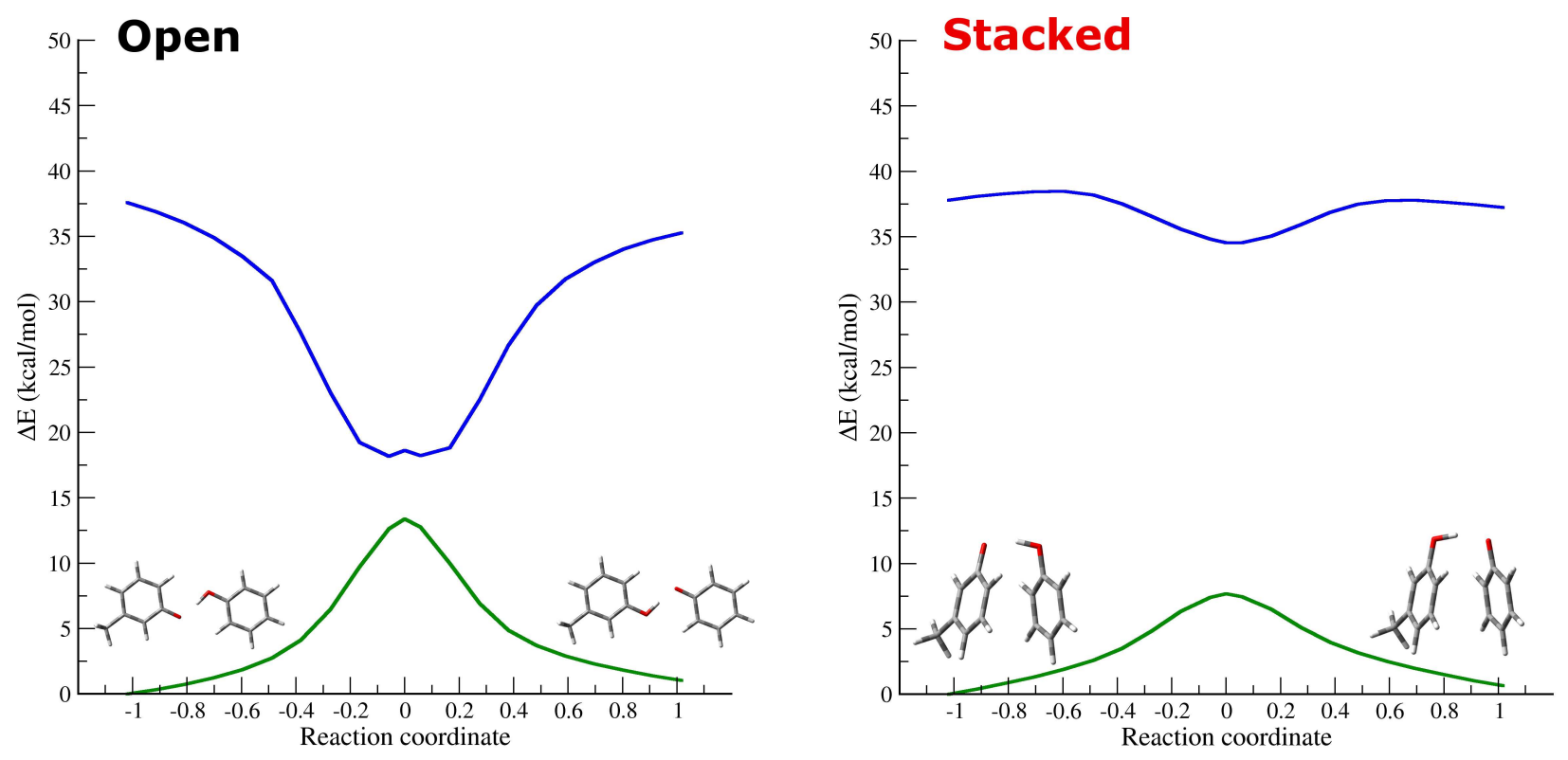

Figure 2: Ground (green line) and first excited (blue line) energy profiles (kcal/mol) along the IRC for the open (left panel) and stacked (right panel) conformations.

\subsection{Electron density based analysis}

The different electronical adiabaticity degree of the open and stacked conformations is now analyzed and described by means of the $\mathrm{D}_{C T}$ electron density based index. Beside the absolute values of $\mathrm{D}_{C T}$, which are dependent on the distance between the two aromatic rings, is very interesting to analyze its derivatives with respect to the reaction coordinate. The computed $\mathrm{D}_{C T}$ values and its derivatives along the IRC path are reported for the two structural arrangements (open and stacked) in Figure 3a and 3b, respectively. The variation of the electronic density between the ground and first excited states is shown for reactants, transition states and products in the lower panel of Figure 3.

As it can be inferred from Figure $3 \mathrm{a}, \mathrm{D}_{C T}$ is minimum at the TS and its derivative is, in turn, zero in this point. This applies for the open and the stacked conformation as well. Moreover, the open arrangement shows larger derivatives in proximity of the transition state region when compared to the stacked one (Figure 3b). As matter of fact, in the former case a very large electron density redistribution between the ground and first excited states occurs in the reactant and product zones, as it can be observed in the lower panel of Figure 

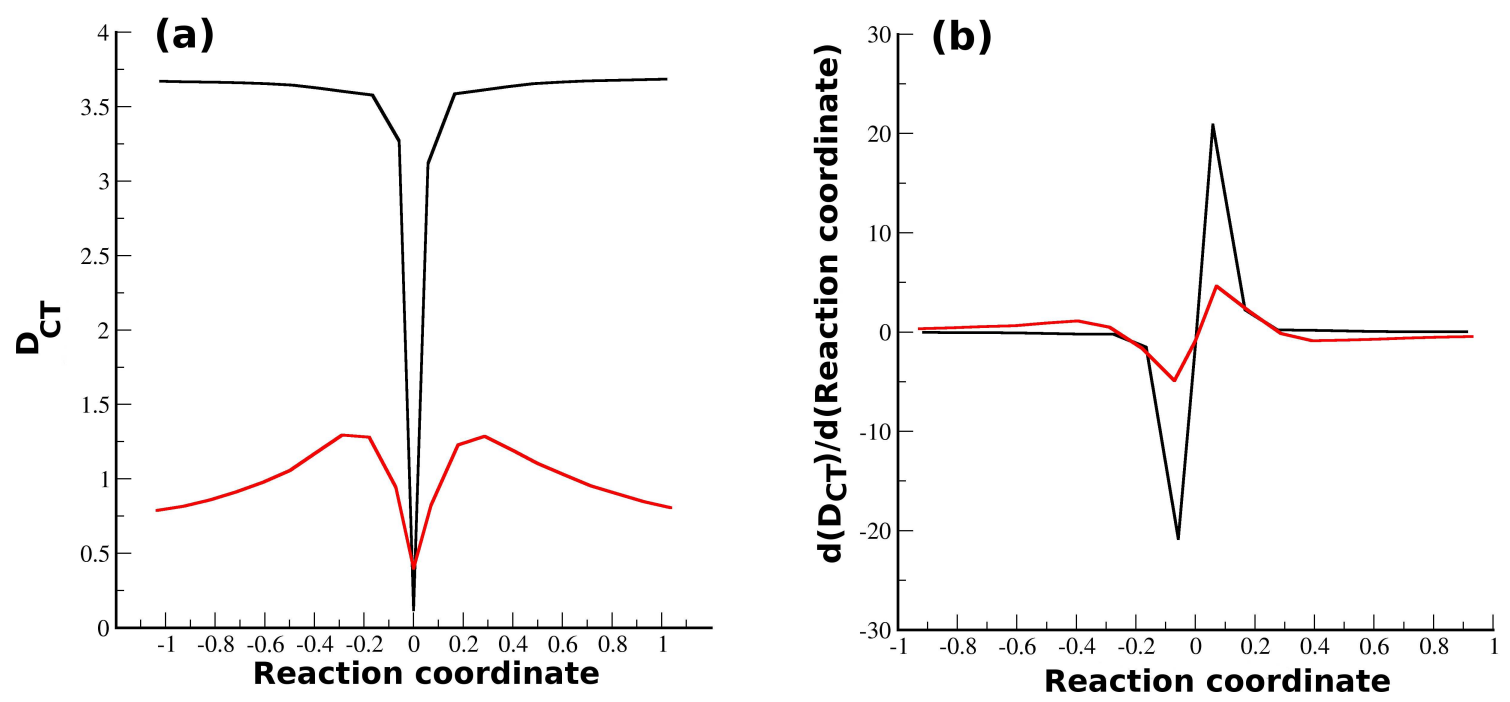

\section{Open}
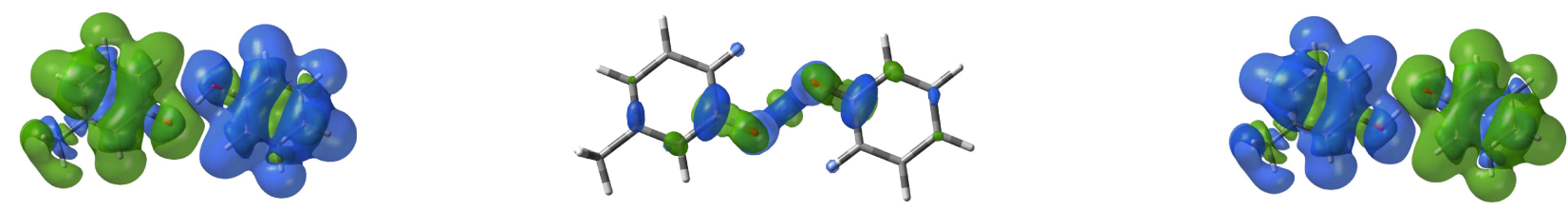

\section{Stacked}

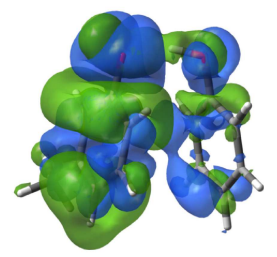

Reactant

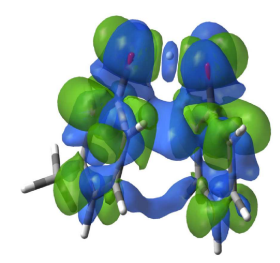

Transition State

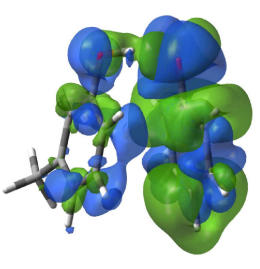

Product

Figure 3: Upper panel: $\mathrm{D}_{C T}(\AA)$ and its derivatives with respect to the reaction coordinate along the IRC path are reported in panels (a) and (b) respectively. Black and red lines refer respectively to the open and the stacked conformations. Lower panel: difference density plots (positive and negative variation of the density is represented in green and blue, respectively) between the ground and first excited state computed for reactans, transitions states and products of the open and stacked geometries.

3. This electronic rearrangement remains constant in these regions while it is very low at the transition state, where the two hydroxyl groups are mainly involved. More closely, a 
depletion of the electron density is observed on the carbon atoms, while it increases on both the phenolic oxygen (Figure 3). The ground-first excited state electronical reorganization can be quantified by the $\mathrm{D}_{C T}$ index, which assumes constant values of about $3.6 \AA$ in both the reactant and product zones, while a value of $0.14 \AA$ is computed at the TS (see Table $\mathrm{S} 2$ ). As a result, $\mathrm{D}_{C T}$ derivatives with respect to the reaction coordinate has its maximum variation in the TS region, where the non-adiabatic coupling is larger, while it is constant in the other zones of the PES.

On the other hand, a different behavior for the stacked dimer can be inferred from Figure 3a. Starting from a value of about $0.7 \AA$ in the reactant region, $\mathrm{D}_{C T}$ gradually increases, reaching a value of about $1 \AA$ before the transition state, where it softly decreases at 0.45 $\AA$. From here, this index starts to rise up to a value of about $1 \AA$ to slowly decrease in the product zone, where it reaches again the value of $0.7 \AA$.

In turn the $\mathrm{D}_{C T}$ derivative slightly grows up when moving from the reactant, while a steeper variation is observed at the transition state. From the density difference plot associated to the ground and first excited state along the reaction coordinate (see Figure S2 in SI) it is clear that the ground-first excited state electronic reorganization increases when going from the reactant to the transition state region. More in detail, the electronic reorganization mainly affects the phenoxyl ring on the reactant structure (see lower panel of Figure 3 and Figure S2). Moving along the reaction coordinate also the other ring gets involved in the electron density redistribution. Inspection of the molecular orbitals involved in the excitation reveals a charge transfer transition from one aromatic ring to the other when moving along the IRC path. This leads, in turn, to the observed $\mathrm{D}_{C T}$ growth and supports also the hypothesis of a HAT mechanism with a small amount of EPT character proposed for the stacked geometry by Harshan and coworkers. ${ }^{35}$ A specular behavior is observed when going from the transition state to the product, while at the TS the electronic rearrangement is minimum and the $\mathrm{D}_{C T}$ falls down. On the other hand, in the stacked geometry the two aromatic rings are both involved in the electronic reorganization, due to their face to face arrangement.

These results suggest that the $\mathrm{D}_{C T}$ index and its variation along the reaction path are able to capture the different electronic adiabaticity degree shown by concerted PCET reactions. 
Indeed, in the case of the open geometry, where an electronically non-adiabatic process occurs, a larger variation of the $\mathrm{D}_{C T}$ index is observed in the transition state zone. More similar the ground and the excited state densities are, lower the $\mathrm{D}_{C T}$ value is, as it happens in highly coupled region of the PES. The low value assumed by the $\mathrm{D}_{C T}$ at the open TS suggests a high similarity between the ground and the excited state electron densities, in accordance with their strong coupling in this region due to the non-adiabaticity degree of the self exchange reaction.

Otherwise, when the reaction is electronically adiabatic, as in the case of the stacked conformation, the electronic coupling is lower at the transition state, and in turn the $\mathrm{D}_{C T}$ derivatives is also smaller in this region.

Finally, the adiabaticity degree of the considered PCET reactions was examined analyzing the first-order non-adiabatic coupling vectors between the ground and the first excited states, and the trend of the root mean square (RMS) of NAC vectors and $\mathrm{D}_{C T}$ along the reaction coordinate was compared.

The root mean square of the cartesian NAC vectors is reported in Figure 4a, while the derivatives of the NAC RMS respect to the reaction coordinate are shown in Figure 4b. The dimensions of the NAC vectors are $[\text { length }]^{-1}$ while the $\mathrm{D}_{C T}$ index is simply a distance. For this reason, we compared the root mean square of the NAC vectors with the inverse of $\mathrm{D}_{C T}, 1 / \mathrm{D}_{C T}$, in Figure 4.

The inspection of Figure 4 shows a very different behavior for the two conformations, in agreement with the CASSCF results reported by Harshan and co-workers. ${ }^{35}$ When an electronically non-adiabatic process occurs (open geometry), ground and excited state are strongly coupled, and the root mean square of the NAC vectors assumes large values in the transition state region. On the other hand, the coupling drastically decreases for the electronically adiabatic proton exchange reaction of the stacked arrangement, and low values of the NAC RMS are observed. Both the conformations show weakly coupled electronic states in the reactant and product zone.

By comparing the variations of the NAC RMS and $1 / \mathrm{D}_{C T}$ along the reaction path is clear that they strictly follow the same trend. Moreover, their derivatives along the reaction coordinate are also in very nice agreement (Figure $4 \mathrm{~b}$ ). These outcomes support and encourage 

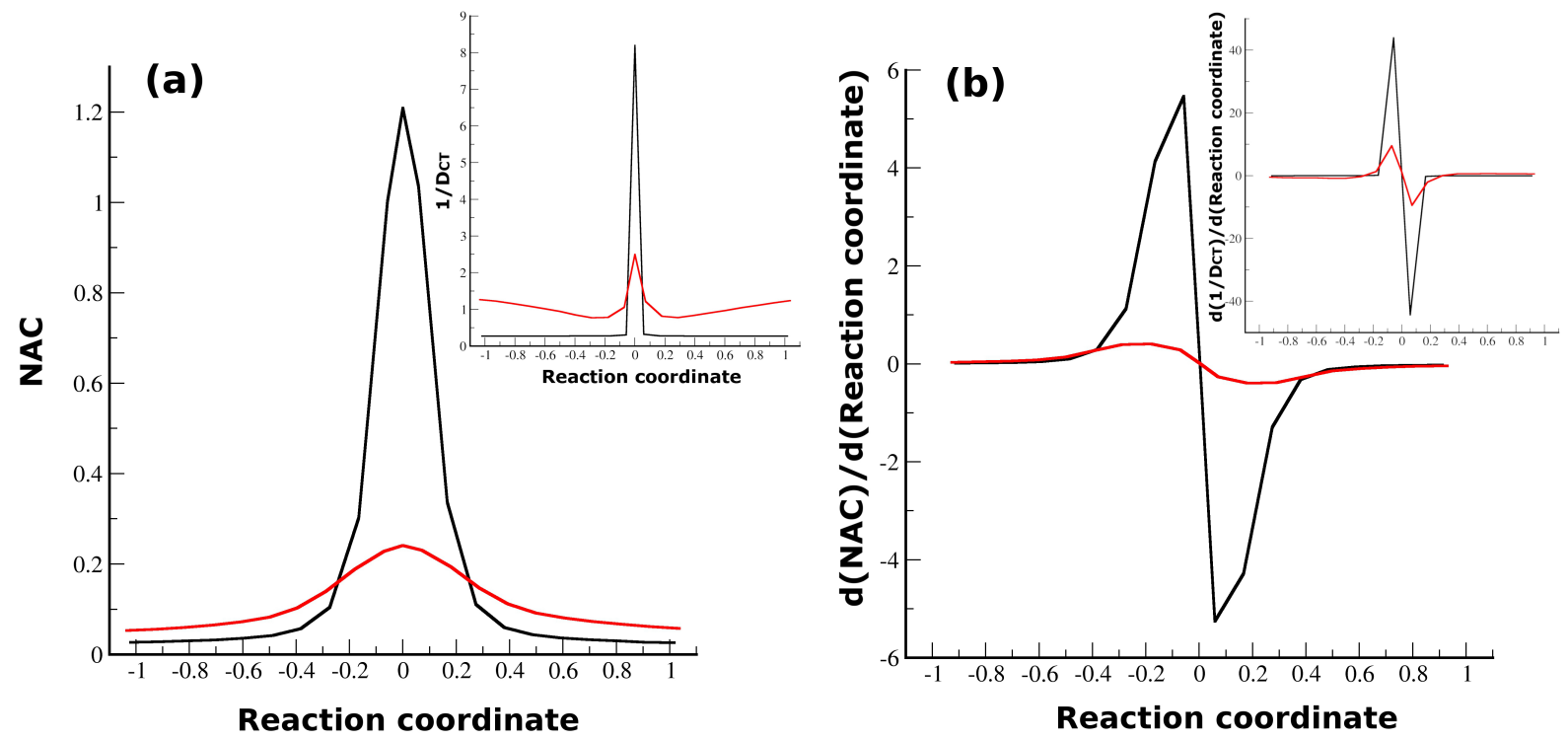

Figure 4: Panel (a) shows the root mean square of the cartesian non-adiabatic coupling vectors $\left(\right.$ bohr $\left.^{-1}\right)$ between the ground and first excited electronic states. The derivatives of the NAC root mean square with respect to the reaction coordinate along the IRC path is reported in panel (b). The inverse of $\mathrm{D}_{C T}, 1 / \mathrm{D}_{C T}\left(\AA^{-1}\right)$, and its derivatives with respect to the reaction coordinate along the IRC path are reported as inset in panel (a) and (b) respectively. Black and red lines refer respectively to the open and the stacked arrangements.

the use of electron density based indexes to describe the electronical adiabaticity degree of PCET reactions.

\section{Conclusions}

In conclusion, a new protocol based on the electron density analysis was successfully applied to recognize the different electronical adiabaticity degree of concerted PCET reactions. The $\mathrm{D}_{C T}$ electron density based index was used to discriminate the HAT and EPT mechanisms shown by the stacked and open conformations of the 3-Methylphenoxyl/phenol system. Our analysis along the reaction coordinate, suggest that this index provides a fair picture of the degree of adiabaticity for the examined cases, following the same behaviour of the root mean square of the non adiabatic coupling vectors between the ground and the first excited states. 
Moreover, a very nice agreement with the conclusions reported by Harshan and co-workers is found.

This rather cheap and smart analysis provides a very promising diagnostic tool to get new insights on complex PCET mechanisms for real systems at low computational cost.

\section{Acknowledgments}

U.R., M.G.C., F.C., F.P. and N.R. gratefully acknowledge funds from Regione Campania (L.R. 5, BRC3179) and Gaussian, Inc. (Wallingford, CT). I.C. acknowledges funding from the H2020 European Research Council (ERC CoG STRIGES n 648558). 


\section{References}

1. M. Wikström, Curr. Opin. Struct. Biol. 8, 480 (1998).

2. H. Michel, Biochemistry 38, 15129 (1999).

3. E. A. Berry, M. Guergova-Kuras, L.-s. Huang, and A. R. Crofts, Annu. Rev. Biochem. 69, 1005 (2000).

4. A. R. Crofts, S. Lhee, S. B. Crofts, J. Cheng, and S. Rose, Biochim. Biophys. Acta, Bioenerg. 1757, 1019 (2006).

5. R. Improta, S. Antonello, F. Formaggio, F. Maran, N. Rega, and V. Barone, J. Phys. Chem. B 109, 1023 (2005).

6. M. Okamura, M. Paddock, M. Graige, and G. Feher, Biochim. Biophys. Acta, Bioenerg. 1458, 148 (2000).

7. M. Saraste, Science 283, 1488 (1999).

8. S. Y. Reece and D. G. Nocera, Annu. Rev. Biochem. 78, 673 (2009).

9. T. J. Meyer, M. H. V. Huynh, and H. H. Thorp, Angew. Chem., Int. Ed. 46, 5284 (2007).

10. R. Cukier, Biochim. Biophys. Acta, Bioenerg. 1655, 37 (2004).

11. N. Madhiri and H. Finklea, Langmuir 22, 10643 (2006).

12. C. Costentin, M. Robert, and J. Savéant, J. Am. Chem. Soc. 128, 4552 (2006).

13. B. Li, J. Zhao, K. Onda, K. Jordan, J. Yang, and H. Petek, Science 311, 1436 (2006).

14. R. I. Cukier and D. G. Nocera, Annu. Rev. Phys. Chem. 49, 337 (1998).

15. J. M. Mayer, Annu. Rev. Phys. Chem. 55, 363 (2004).

16. A. Migliore, N. F. Polizzi, M. J. Therien, and D. N. Beratan, Chem. Rev. 114, 3381 (2014). 
17. D. R. Weinberg, C. J. Gagliardi, J. F. Hull, C. F. Murphy, C. A. Kent, B. C. Westlake, A. Paul, D. H. Ess, D. G. McCafferty, and T. J. Meyer, Chem. Rev. 112, 4016 (2012).

18. M. H. V. Huynh and T. J. Meyer, Chem. Rev. 107, 5004 (2007).

19. S. Hammes-Schiffer, Chem. Rev. 110, 6937 (2010).

20. S. Hammes-Schiffer, Energy Environ. Sci. 5, 7696 (2012).

21. S. Hammes-Schiffer and A. A. Stuchebrukhov, Chem. Rev. 110, 6939 (2010).

22. S. Hammes-Schiffer, J. Am. Chem. Soc. 137, 8860 (2015).

23. S. Hammes-Schiffer, Chem. Phys. Chem. 3, 33 (2002).

24. C. Costentin, Chem. Rev. 108, 2145 (2008).

25. J. M. Mayer and I. J. Rhile, Biochim. Biophys. Acta, Bioenerg. 1655, 51 (2004).

26. S. Hammes-Schiffer, E. Hatcher, H. Ishikita, J. H. Skone, and A. V. Soudackov, Coord. Chem. Rev. 252, 384 (2008).

27. O. Tishchenko, D. G. Truhlar, A. Ceulemans, and M. T. Nguyen, J. Am. Chem. Soc. 130, 7000 (2008).

28. M. D. Symes, Y. Surendranath, D. A. Lutterman, and D. G. Nocera, J. Am. Chem. Soc. 133, $5174(2011)$.

29. C. J. Gagliardi, B. C. Westlake, C. A. Kent, J. J. Paul, J. M. Papanikolas, and T. J. Meyer, Coord. Chem. Rev. 254, 2459 (2010).

30. M. D. Karkas, E. V. Johnston, O. Verho, and B. Kermark, Acc. Chem.Res. 47, 100 (2013).

31. M. J. Llansola-Portoles, R. E. Palacios, D. Gust, T. A. Moore, and A. L. Moore, in From Molecules to Materials (Springer, 2015), pp. 71-98.

32. D. Gust, T. A. Moore, and A. L. Moore, Faraday discuss. 155, 9 (2012). 
33. J. D. Megiatto, A. Antoniuk-Pablant, B. D. Sherman, G. Kodis, M. Gervaldo, T. A. Moore, A. L. Moore, and D. Gust, Proc.Natl.Acad.U.S.A. 109, 15578 (2012).

34. S. Berardi, S. Drouet, L. Francàs, C. Gimbert-Suriñach, M. Guttentag, C. Richmond, T. Stoll, and A. Llobet, Chem.Soc.Rev. 43, 7501 (2014).

35. A. K. Harshan, T. Yu, A. V. Soudackov, and S. Hammes-Schiffer, J. Am. Chem. Soc. 137, 13545 (2015).

36. J. H. Skone, A. V. Soudackov, and S. Hammes-Schiffer, J. Am. Chem. Soc. 128, 16655 (2006).

37. A. Sirjoosingh and S. Hammes-Schiffer, J. Phys. Chem. A 115, 2367 (2011).

38. R. Send and F. Furche, J. Chem. Phys. 132, 044107 (2010).

39. V. Chernyak and S. Mukamel, J. Chem. Phys. 112, 3572 (2000).

40. D. B. Lingerfelt, D. B. Williams-Young, A. Petrone, and X. Li, J. Chem. Theory Comput. 12, 935 (2016).

41. M. G. Chiariello, U. Raucci, F. Coppola, and N. Rega, Phys. Chem. Chem. Phys. 21, 3606 (2019).

42. M. Barbatti, WIREs: Computat. Mol. Sci. 1, 620 (2011).

43. J. D. Coe, M. T. Ong, B. G. Levine, and T. J. Martínez, J. Phys. Chem. A 112, 12559 (2008).

44. W. Domcke, D. R. Yarkony, and H. Köppel, Conical Intersections: Theory, Computation and Experiment, vol. 17 (World Scientific, 2011).

45. N. C. Handy and A. M. Lee, Chem. Phys. Lett. 252, 425 (1996).

46. D. G. Truhlar and C. A. Mead, Phys. Rev. A 68, 032501 (2003).

47. J. C. Tully, J. Chem. Phys. 93, 1061 (1990). 
48. J. C. Tully, in Modern Methods for Multidimensional Dynamics Computations in Chemistry (World Scientific, 1998), pp. 34-72.

49. A. Petrone, D. B. Lingerfelt, D. B. Williams-Young, and X. Li, J. Phys. Chem. Lett. 7, 4501 (2016).

50. J. L. Stein, M. I. Steimle, M. W. Terban, A. Petrone, S. J. Billinge, X. Li, and B. M. Cossairt, Chem. Mater. 29, 7984 (2017).

51. A. Petrone, J. J. Goings, and X. Li, Phys. Rev. B 94, 165402 (2016).

52. R. Spezia, I. Burghardt, and J. T. Hynes, Mol. Phys. 104, 903 (2006).

53. J. P. Malhado, R. Spezia, and J. T. Hynes, Int. J. Quant. Chem. 113, 296 (2013).

54. M. J. Bearpark, M. A. Robb, and H. B. Schlegel, Chem. Phys. Lett. 223, 269 (1994).

55. G. Donati, D. B. Lingerfelt, A. Petrone, N. Rega, and X. Li, J. Phys. Chem. A 120, 7255 (2016).

56. C. Adamo, T. Le Bahers, M. Savarese, L. Wilbraham, G. García, R. Fukuda, M. Ehara, N. Rega, and I. Ciofini, Coord. Chem. Rev. 304, 166 (2015).

57. T. Le Bahers, C. Adamo, and I. Ciofini, J. Chem. Theory Comput. 7, 2498 (2011).

58. M. Savarese, P. A. Netti, C. Adamo, N. Rega, and I. Ciofini, J. Phys. Chem. B 117, 16165 (2013).

59. L. Wilbraham, M. Savarese, N. Rega, C. Adamo, and I. Ciofini, J. Phys. Chem. B 119, 2459 (2014).

60. M. Savarese, U. Raucci, P. A. Netti, C. Adamo, N. Rega, and I. Ciofini, Theor. Chem. Acc. 135, 211 (2016).

61. M. Savarese, U. Raucci, R. Fukuda, C. Adamo, M. Ehara, N. Rega, and I. Ciofini, J. Comput. Chem. 38, 1084 (2017).

62. M. G. Chiariello and N. Rega, J. Phys. Chem. A 122, 2884 (2018). 
63. F. Maschietto, J. Sanz García, M. Campetella, and I. Ciofini, J. Comput. Chem. 40, 650 (2019).

64. A. Petrone, P. Cimino, G. Donati, H. P. Hratchian, M. J. Frisch, and N. Rega, J. Chem. Theory Comput. 12, 4925 (2016).

65. G. Donati, A. Petrone, P. Caruso, and N. Rega, Chemical Science 9, 1126 (2018).

66. M. Savarese, U. Raucci, C. Adamo, P. A. Netti, I. Ciofini, and N. Rega, Phys. Chem. Chem. Phys. 16, 20681 (2014).

67. M. Ehara, R. Fukuda, C. Adamo, and I. Ciofini, J. Comput. Chem. 34, 2498 (2013).

68. R. G. Parr and W. Yang, Density-Functional Theory of Atoms and Molecules, vol. 16 (Oxford university press, 1989).

69. R. E. Stratmann, G. E. Scuseria, and M. J. Frisch, J. Chem. Phys. 109, 8218 (1998).

70. E. Runge and E. K. Gross, Phys. Rev. Lett. 52, 997 (1984).

71. Y. Zhao and D. Truhlar, Theor. Chem. Acc. 120, 215 (2008).

72. H. P. Hratchian and H. B. Schlegel, J. Chem. Phys. 120, 9918 (2004).

73. C. Dykstra, G. Frenking, K. Kim, and G. Scuseria, Theory and Applications of Computational Chemistry: the first forty years (Elsevier, 2011).

74. H. Hratchian and H. Schlegel, J. Chem. Theory Comput. 1, 61 (2005).

75. T. Yanai, D. P. Tew, and N. C. Handy, Chem. Phys. Lett. 393, 51 (2004).

76. D. Jacquemin, E. A. Perpete, G. E. Scuseria, I. Ciofini, and C. Adamo, J. Chem. Theory Comput. 4, 123 (2008).

77. M. J. Frisch, G. W. Trucks, H. B. Schlegel, G. E. Scuseria, M. A. Robb, J. R. Cheeseman, G. Scalmani, V. Barone, G. A. Petersson, H. Nakatsuji, et al., Gaussian16 Revision A.03 (2016), gaussian Inc. Wallingford CT. 